\title{
Impact of the memory hierarchy on shared memory architectures in multicore programming models
}

\author{
Rosa M. Badia, Josep M. Perez, Eduard Ayguadé and Jesus Labarta \\ Barcelona Supercomputing Center and Universitat Politècnica de Catalunya \\ Barcelona, SPAIN \\ \{rosa.m.badia, josep.m.perez, eduard.ayguade, jesus.labarta\}@bsc.es
}

\begin{abstract}
Many and multicore architectures put a big pressure in parallel programming but gives a unique opportunity to propose new programming models that automatically exploit the parallelism of these architectures. OpenMP is a very well known standard that exploits parallelism in shared memory architectures. SMPSs has recently been proposed as a task based programming model that exploits the parallelism at the task level and takes into account data dependencies between tasks. However, besides parallelism in the programming, the memory hierarchy impact in many/multi core architectures is a feature of large importance. This paper presents an evaluation of these two programming models with regard to the impact of different levels of the memory hierarchy in the duration of the application. The evaluation is based on tracefiles with hardware counters on the execution of a memory intensive benchmark in both programming models.

Keywords: SMP Superscalar, programming models for multicore, task scheduling, locality exploitation
\end{abstract}

\section{Introduction}

The new trends in computer fabrication have evolved towards machines at all levels (from customer to HPC large systems) with multicore homogeneous or heterogeneous chips. For this reason it is now, more than ever, true that there is a need for parallel programming models that enable to easily exploit the possibilities of these chips. There are several programming models that have been proposed with this objective. OpenMP [1] is a very well known standard that exploits parallelism in shared memory architectures. The recent version 3.0 [2] extends its functionality to include the support of task level parallelism. In a similar way, SMP Superscalar (SMPSs) [3] has recently been proposed as a task based programming model that exploits the parallelism at the task level and takes into account data dependencies between tasks. Both will be the focus in this paper. While previous studies [3], [4] have focused more on the performance achieved by the corresponding runtimes, this paper presents an evaluation of these two programming models with regard to the impact of different levels of the memory hierarchy in the duration of the application tasks. The evaluation is based on tracefiles with hardware counters on the execution of a set of benchmarks in both programming models.

The paper structure is as follows: section 2 outlines the main characteristics of SMPSs and OpenMP, section 3 outlines some related work, section 4 presents experimental results with the STREAM benchmark, section 5 outlines a new mechanism to further improve the locality exploitation in SPMSs and finally section 6 concludes the paper.

\section{Task Based Programming Models}

Since the focus of this paper is SMPSs and OpenMP the next two subsections present more detailed summary of these two programming models.

\subsection{SMP Superscalar}

Star Superscalar (StarSs) is a family of programming models (SMPSs [3], CellSs [5] and others). This programming model is inspired by the behavior of superscalar processors that are able to execute more than one instruction during a clock cycle by simultaneously dispatching multiple instructions to redundant functional units on the processor. For StarSs the unit of execution is not the instruction but a task, and a task is a function in the code without collateral effects (only variables and parameters are accessed) and with enough grain (this may depend on the final target architecture where StarSs is implemented, and can be tuned by applying blocking for example). The basic idea behind StarSs is that tasks are defined by a sequential program and at runtime a Directed Acyclic Graph (DAG) is built where each of the nodes of the DAG represent a task and edges represent data precedences that must be respected and that are automatically detected by the runtime library.

Tasks are identified by pragma annotations of the type:

\#pragma css task input (in_var1, in_var2)

output (out_var) inout (in_out_var)

where input, output and inout denotes the direction of the parameters of the task. The case of study in this paper is SMP Superscalar (SMPSs), that targets homogeneous multicore processors or shared memory machines. The current 
runtime is implemented in such a way that for an application that runs with $\mathrm{N}$ threads, the first thread (main thread) executes both the main sequential program and tasks (if time is left) and the other threads (worker threads) only execute tasks. The scheduling of each task is determined partially by the order in which the tasks are called, partially by the data dependences, and partially by the thread that executes the last predecessor of the task, with the objective of scheduling in the same core dependant tasks. This favors locality exploitation since a dependence denotes that the predecessor task is writing a piece of data that is going to be read by the successor task.

There exist $\mathrm{N}+1$ ready lists, one main ready list and one additional for each of the threads (including one for the main thread). Whenever a task is generated, the runtime looks for data dependences between the new task and former tasks. If these data dependences exist, the task is inserted in the DAG. However, if the task does not hold any data dependency, then it is ready for execution and it is inserted in the main ready list. The behavior of the worker threads is that they always consume tasks from their ready list unless this is empty, then they consume tasks from the main ready list, and if this is also empty the threads will try to steal tasks from the ready lists of other workers. Threads consume tasks from their own ready list in LIFO order, consume tasks from the main ready list and from other threads' list in FIFO order. Whenever a task is finished, the threads update the corresponding data structures in the DAG and if the task completion has released all the remaining data dependences of one or more tasks, those are inserted in the thread ready list.

\subsection{OpenMP}

OpenMP was born in the 1990s with the objective of bringing a standard to the different directive languages defined by a community of vendors. Thanks to a set of characteristics: simplicity of the interface, use of a shared memory model, and use of loosely-coupled directives to express the parallelism of a program, it is very well-accepted today. OpenMP is based on the insertion of directives in the sequential source code that give hints to the runtime library about the existent parallelism in the application. The OpenMP pragma annotation denoting a parallel loop is as follows:

\#pragma omp parallel for

Version 3.0 of OpenMP includes a tasking model that fills a gap with regard the ways of expressing parallelism in an application. With the new OpenMP directives, the programmers can identify units of independent work (tasks), leaving the decision to how and when to execute them to the runtime system. This gives the programmers a way of expressing patterns of concurrency that do not match the worksharing constructs defined in the OpenMP 2.5 specification.

\section{Related work}

One of the task-based programming models is Cilk [6], a general-purpose programming language designed for multithreaded parallel programming. In Cilk, the programmer is responsible of exposing the application parallelism, identifying sections of the code (tasks) that can safely be executed in parallel. Tasks are invoked with the spawn keyword and the sync keyword is used to wait until all previously spawned tasks have completed. Cilk supports recursivity at the task level (tasks generate new tasks) but does not support automatic data dependence detection between them. Therefore, data dependences have to be controlled by the programmer with the help of the sync keyword. The runtime, in particular the scheduler, decides how to actually divide the work between processors. The work-stealing approach followed by the Cilk scheduler has been designed in such a way that naturally exploits the existent data locality, in particular for the recursive tasks.

Cilk initially only supported parallel tasks, however, Cilk++ also supports parallel loops. OpenMP evolution is just the opposite: initially supported parallel loops, while the last version 3.0 also supports parallel tasks. Both systems support also recursivity at the task level. SMPSs does not support parallel loops, but supports task parallelism, although does not support the recursivity at the task level. The main difference between SMPSs and the previous two approaches is that SMPSs automatically detects the task dependences building a task DAG.

Besides, there have been steps towards the integration of task precedence [7] and task dependence [8] in OpenMP.

With regard to related work on studies the behavior of applications in ccNUMA shared memory systems, [9] presents the results of cache and memory performance studies on an SGI Altix 350. In [10] the authors present an study of the performance obtained (with relation to the ccNUMA memory) in a Sun Fire Server. The paper also proposes son performance tunings that improve up to $30 \%$ the application performance. In [11] the authors present the evaluation of the SARC programming model on the Cell/BE architecture using the benchmarks STREAM and RandomAccess.

\section{Experiments}

The first part of the paper is focused in the description of the STREAM benchmark from the HPC Challenge collection [12]. We present alternative implementations to the original OpenMP one and an SMPSs implementation of this benchmark and an analysis of the results.

\subsection{STREAM implementation in OpenMP}

STREAM [12] is a simple synthetic benchmark program that measures sustainable memory bandwidth and the corresponding computation rate for a simple vector kernel. 


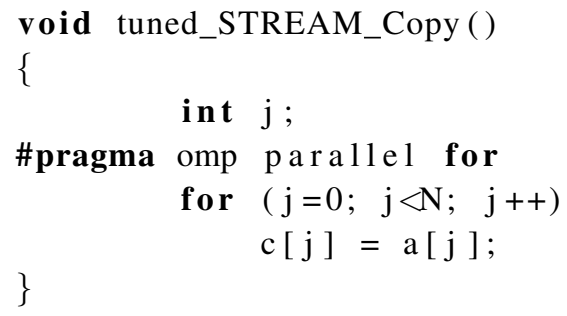

Figure 1: Original copy function in STREAM

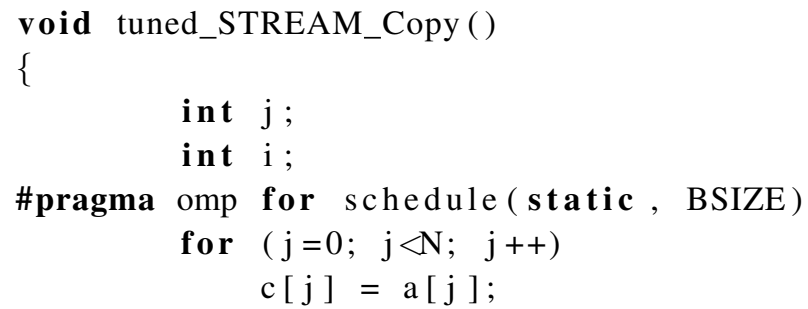

Figure 2: Modified OpenMP version of copy function in STREAM

The original STREAM implementation already considers an OpenMP parallelization of four routines ((Copy, Scale, Add and Triad), as it is shown in figure 1 for the original Copy function in STREAM.

For the purposes of this paper we have considered an alternative implementation as shown in figure 2, that mimics the behavior of the SMPSs implementation explained in the next section. This version statically distributes chunks of BSIZE iterations to each of the threads.

Additionally, a parallel pragma is inserted in the main program, as shown in figure 3. Besides, a dynamically scheduled version as shown in 4 is also considered.

The original benchmark includes in its prelude a par-

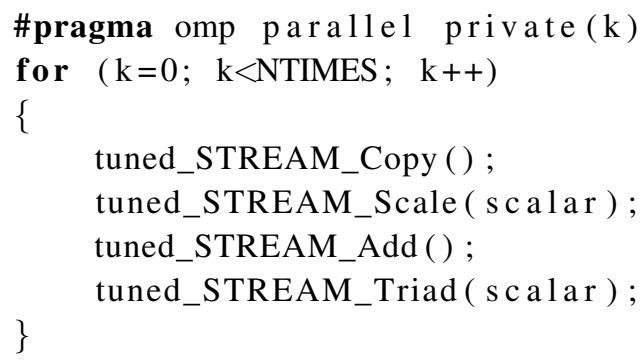

Figure 3: Modified OpenMP version of main program in STREAM

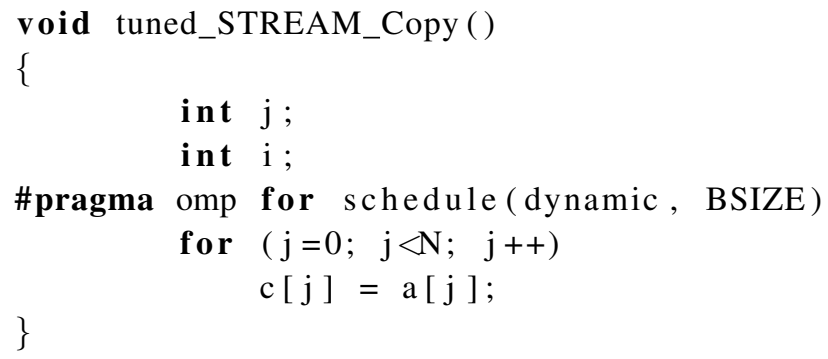

Figure 4: OpenMP version of copy function in STREAM with dynamic scheduling

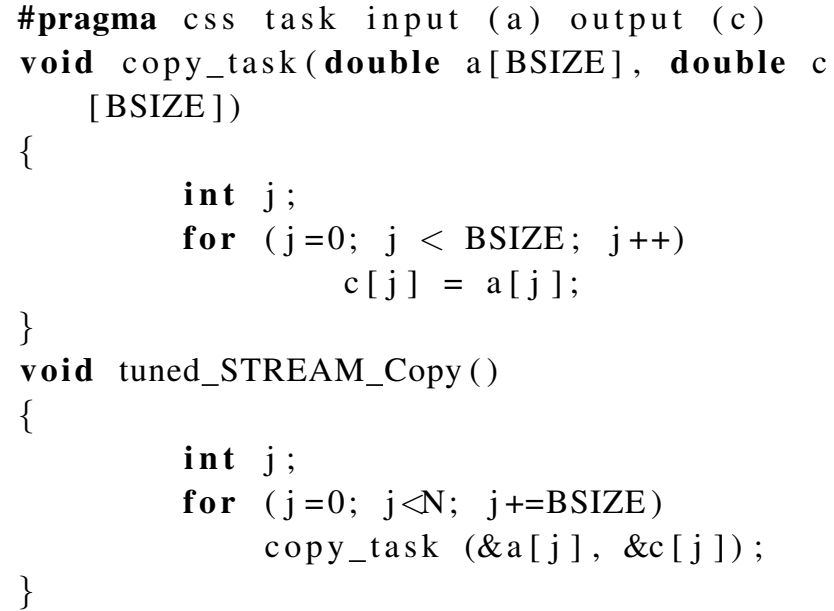

Figure 5: Copy function in STREAM with SMPSs

allelized loop where the data arrays are initialized. This initialization has been slightly modified to consider also the static and dynamic scheduling of BSIZE chunks of iterations. To compare with SMPSs, we will also include results where the initialization is done sequentially.

\subsection{STREAM implementation in SMPSs}

For the SMPSs version, we encapsulated chunks of consecutive iterations of the loops of functions Copy, Scale, Add and Triad into SMPSs tasks. Figure 5 shows the code changes for function Copy.

The STREAM main code is a loop that calls the different functions. To mimic the same behavior as in the original benchmark, we have initially inserted explicit barrier synchronizations between the calls to Copy, Scale, ... as shown in figure 6. In this first version, all Copy tasks of one iteration are performed first, then all Scale tasks, etc. This not only mimics the original STREAM benchmark, but also models how a naive task scheduling algorithm will schedule 


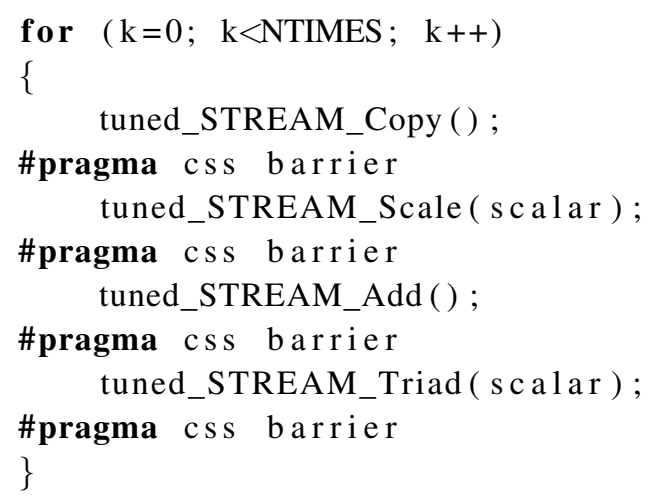

Figure 6: Main loop of STREAM for SMPSs version

$$
\begin{aligned}
& \mathrm{c}[\mathrm{j}]=\mathrm{a}[\mathrm{j}] ; \quad / / \text { Copy } \\
& \mathrm{b}[\mathrm{j}]=\mathrm{scalar} * \mathrm{c}[\mathrm{j}] ; / / \text { Scale } \\
& \mathrm{c}[\mathrm{j}]=\mathrm{a}[\mathrm{j}]+\mathrm{b}[\mathrm{j}] ; / / \text { Add } \\
& \mathrm{a}[\mathrm{j}]=\mathrm{b}[\mathrm{j}]+\mathrm{scalar} * \mathrm{c}[\mathrm{j}] ; / / \text { Triad }
\end{aligned}
$$

Figure 7: Actual operations in STREAM for each element of the array

tasks in SMPSs. Such a naive scheduler will have a single ready list and the ready tasks will be inserted in this list as soon as all the data dependences are released, and consumed by the threads in FIFO order.

However, since SMPSs automatically detect the dependences between the tasks, another version is possible, eliminating all the barrier synchronizations. This somehow perverts the original objectives of the benchmark, but enables us to measure how the current scheduling implemented in the SMPSs runtime exploits the locality of the application. If we look at the basic code executed for each element of the vector we have the code listed in figure 7 .

In this code we can see that the data dependences define the following task precedence inside the iterations Copy Scale - Add - Triad, and additionally between iterations, a task precedence between the Triad of one iteration is defined with the Copy task of the next iteration. Therefore, with the scheduling strategy currently implemented in SMPSs, for a chunk of the array all the tasks operating on this chunk can be executed in the same core, exploiting the locality of the benchmark.

Additionally, to mimic the parallel initialization of data that the OpenMP version is able to do, we have encapsulated the initialization of the data arrays in a task (each task initializes a chunk of BSIZE elements of the arrays).

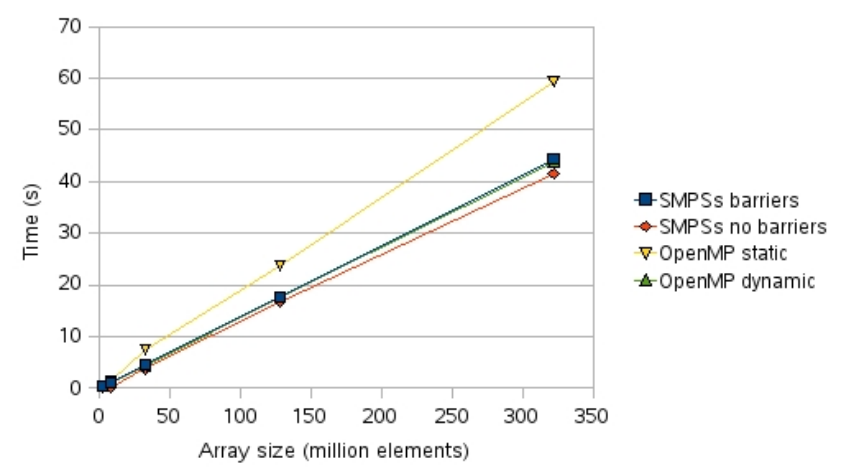

Figure 8: Execution time of the STREAM benchmark with sequential initialization ( 8 processors)

\subsection{Execution environment}

The results presented in this section have been executed in an SGI Altix 4700 at BSC with 128 core (32 nodes, with 2 dual-core processors) and a total of 1TB of memory. We have run SMPSs versions of the experiments with SMPSs version 2.0 and extracted Paraver [13] tracefiles with hardware counters (extracted with the support of PAPI library version 3.5.0). The OpenMP versions have been compiled with the native ICC compiler, version 10.0. This compiler was also used as back-end compiler for the SMPSs versions.

\subsection{Versions' Comparison}

The first experiments we have run consisted in the measurement of the execution time of all the aforementioned versions. We ran the different examples changing the array size, from 2 million elements to 321.93 million elements (this is not an accidental number, since with this size the total amount of memory used is $7368.4 \mathrm{MB}$ which is roughly the $90 \%$ of memory available by each core of this machine). With the objective of analyzing the impact of enlarging the size of the chunks of data assigned to each thread (or task), the chunks' size used is the $1 \%$ of the total size of the array. We have also changed the number of processors, running the experiments with 8,16 and 32 processors.

Figures 8, 9, and 10 show the comparison of the two OpenMP versions (static and dynamic scheduling) against the two SMPSs versions (with and without barriers) when doing the data arrays are sequentially initialized.

When using 8 processors, the OpenMP with static scheduling has the worst behavior, while the OpenMP with dynamic scheduling and SMPSs with barriers perform equally and the SMPSs without barrier slightly outperforms the previous. With 16 processors, the results are very similar, with the difference that now the OpenMP version with dynamic scheduling is a bit better to the SMPSs version with 


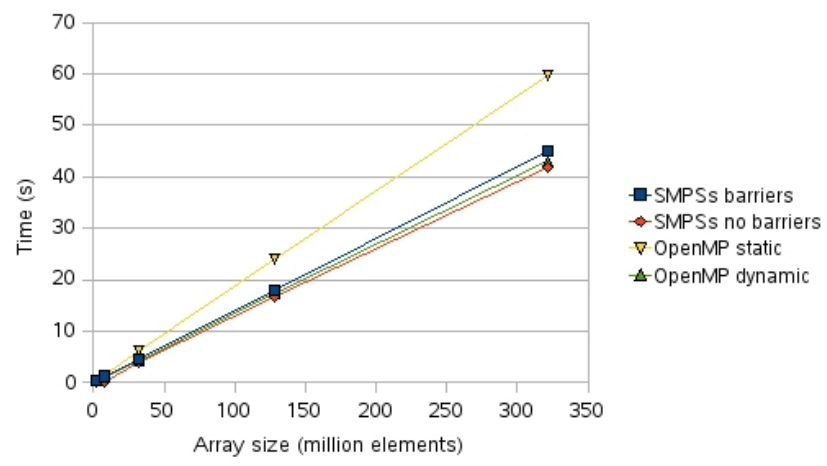

Figure 9: Execution time of the STREAM benchmark with sequential initialization (16 processors)

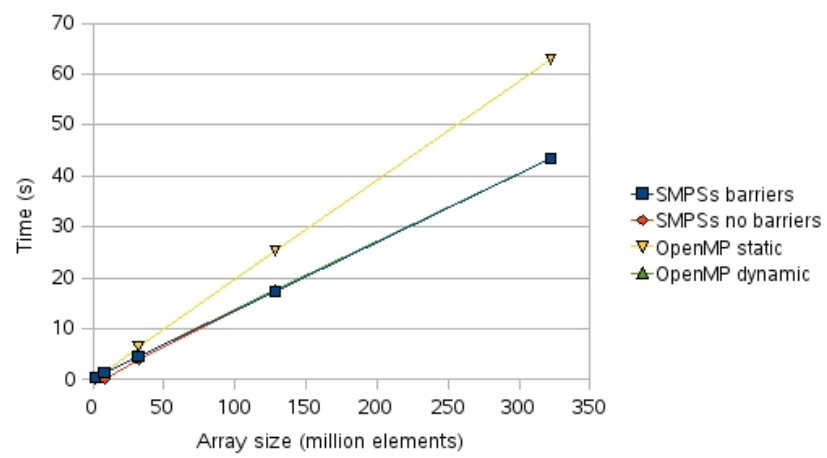

Figure 10: Execution time of the STREAM benchmark with sequential initialization (32 processors)

barriers. With 32 processors, although it is not clearly observable in the chart, besides the OpenMP version with static scheduling that gets the worst results, the rest of versions have the same behavior. Another important observation from these charts is that no improvement in the total execution time is observed when using more processors (none of the cases scale with the number of processors).

Figures 11, 12, and 13 show the comparison of the two OpenMP versions (static and dynamic scheduling) against the two SMPSs versions (with and without barriers) when doing a parallel initialization of the data arrays.

When the arrays are initialized in parallel the situation changes since the physical memory in the SGI Altix is by default allocated on a first touch basis. With 8 processors, the chart looks similar to the situation when using sequential initialization. However, the situation with 16 and 32 processors looks very different, with the OpenMP with static scheduling improving a lot and the OpenMP with dynamic scheduling behaving worst when increasing the number of processors. We would like to stress here that the SMPSs version without barriers shows a more stable behavior in all the cases, being the best or almost the best in all cases.

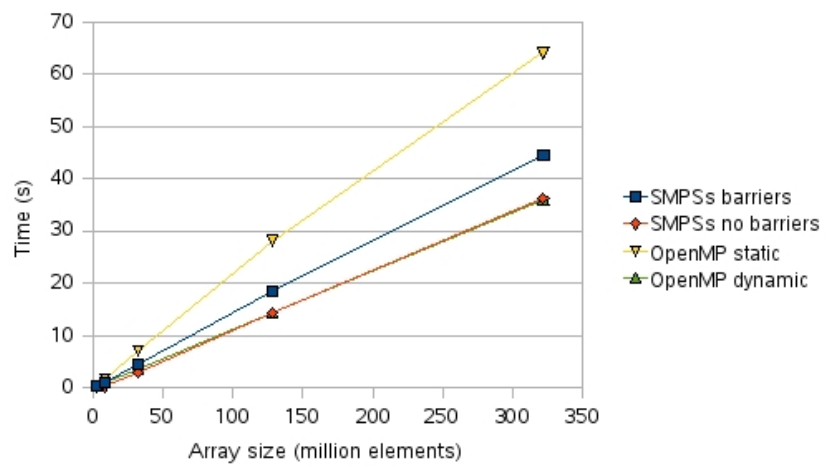

Figure 11: Execution time of the STREAM benchmark with parallel initialization ( 8 processors)

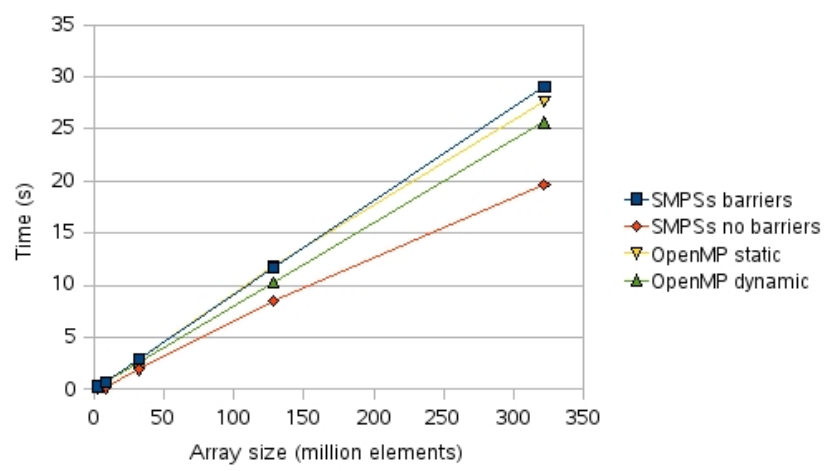

Figure 12: Execution time of the STREAM benchmark with parallel initialization (16 processors)

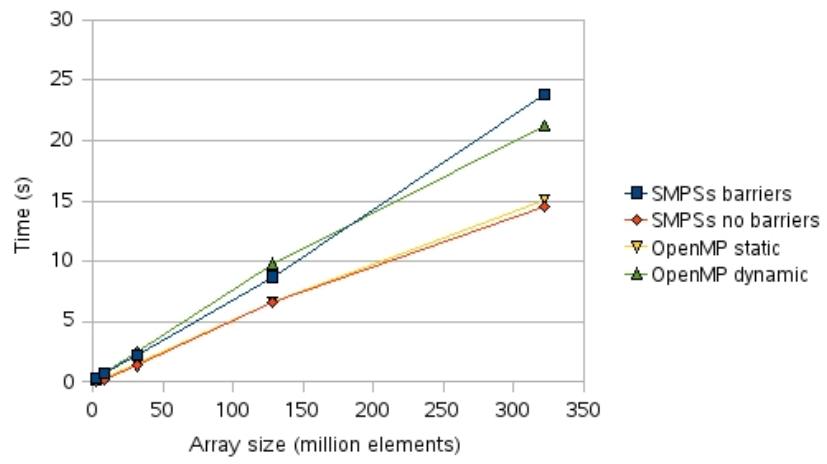

Figure 13: Execution time of the STREAM benchmark with parallel initialization (32 processors) 

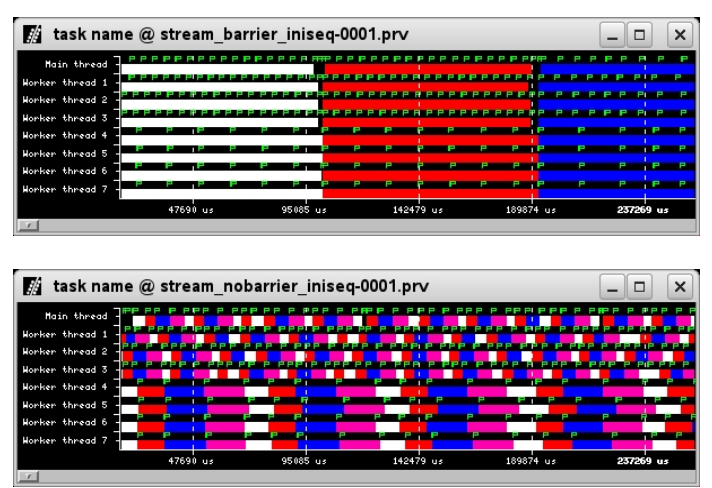

Figure 14: Tasks' scheduling in the SMPSs versions

\subsection{Analysis of the results}

This section reports more in-depth analysis of the tracefiles. The first analysis is done by comparing the four cases when the data arrays are sequentially initialized. The objective of these first analysis is to understand why any of these versions is actually scaling with the number of processors. The analysis is done comparing traces with 8 , 16 and 32 processors.

Regarding the scheduling of the SMPSs versions when analyzing the traces it is observed that in the version with barriers all tasks of a type are executed one after the other to preserve the barriers but in the version without barriers tasks of different type are interleaved, preserving the datadependences and exploiting the locality of the chunks of data. This is shown in figure 14: the $\mathrm{x}$-axis represents the timeline and each line corresponds to one thread. Different colors correspond to different tasks (the small green flags indicate the begin of a new task).

We first had a look to the average time to execute each of the tasks in the different versions. This time is different for each of the tasks' type, as can be observed in figure 15. For this case, with a relatively small data array size, the case with barriers shows also a deviation in time between the first four threads and the other four. This deviation is not observed in the version without barriers since the locality is overall better exploited. This is due to the fact that the arrays are allocated and initialized in the sequential section of the benchmark by the first thread. Therefore, we can assume that the threads $1-4$ are located in the first node and threads 5-8 in another node.

However, when analyzing the traces with a larger data array size, this difference between the version with barriers and without barriers is less evident. This can be observed in figure 16 for the SMPSs versions, when a array size of 32 million of elements is used.

To understand this difference in task time we had a look to the L3 and TLB miss ratio of the different cases. Although these ratios did not show significant differences,
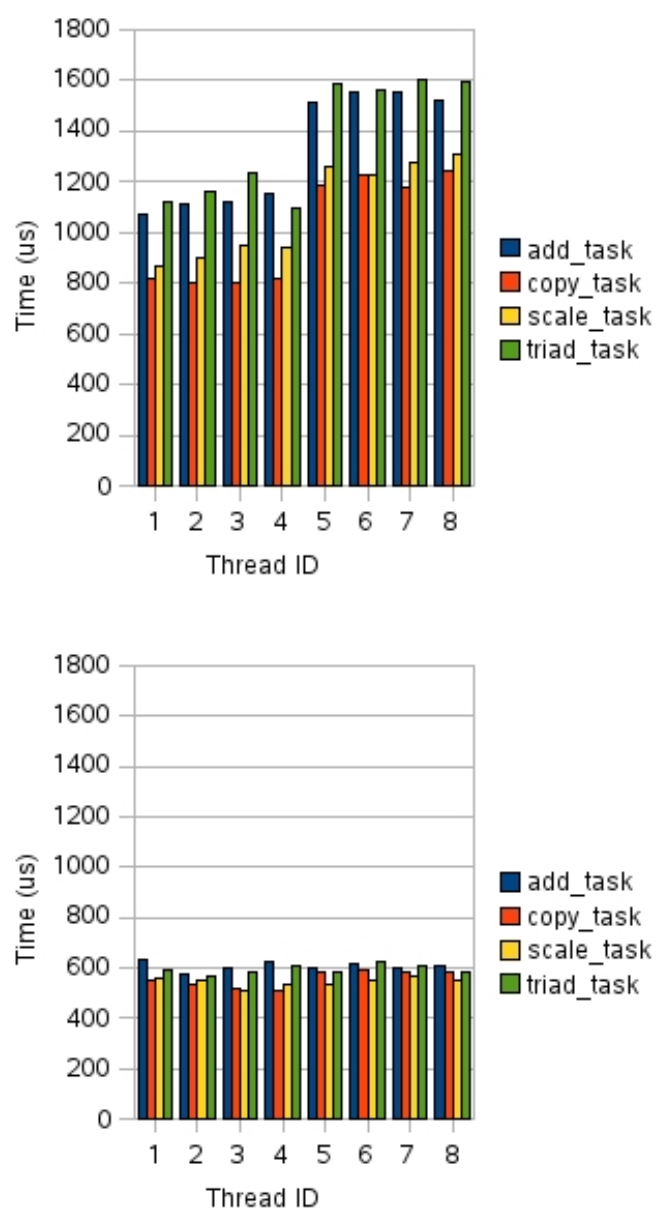

Figure 15: Average time per task and thread, with (top) and without barriers (bottom), using 8 processors and data array size of 2 million elements

the bandwidth obtained by each of the threads in the different cases reflects the same difference, as can be seen in figure 17. In this figure, we see for the SMPSs version without barriers, how the bandwidth with memory varies when we run the benchmark with 8,16 and 32 processors. The figure shows not only the differences between the bandwidth obtained in the first 4 processors (located in the first node) and the others, but also how this difference is each time larger. In the case of 32 processors, the average bandwidth in the processors 4-31 is only $58.8 \mathrm{MB} / \mathrm{s}$ in this case. For the rest of the examples (SMPSs with barriers and OpenMP) the behaviour is similar and we do not show the results due to space and redundancy reasons. Clearly, the fact that the data arrays are initialized in one node creates a bottleneck in this node, and therefore in these systems it is a good idea to distribute the data initialization.

Another interesting fact that we wanted to understand from figures $11-13$ is the behavior of the OpenMP 


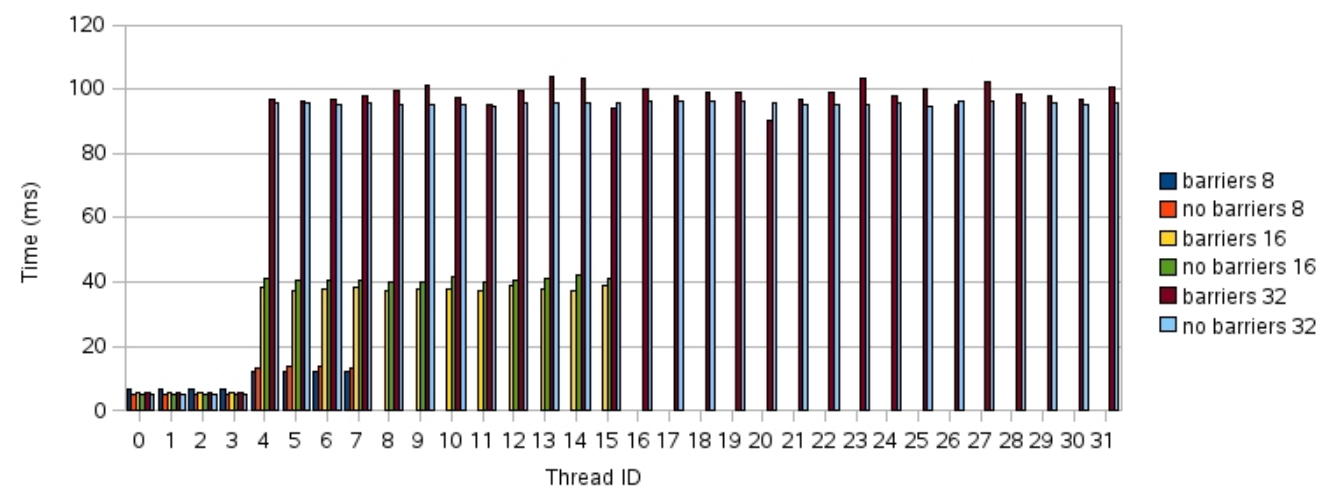

Figure 16: Average execution time of tasks per thread with SMPSs

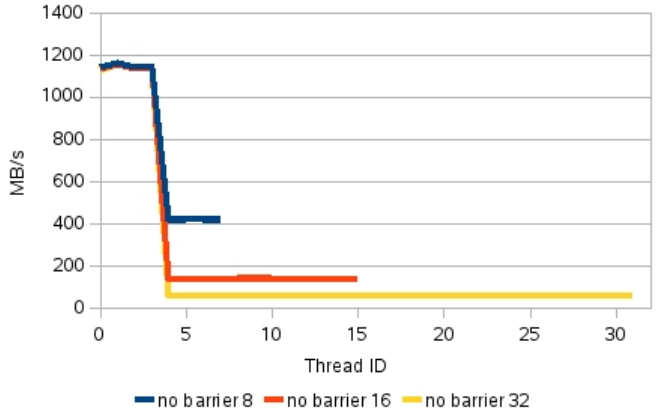

Figure 17: Bandwidth with memory for the SMPSs version without barriers

static version. It is surprising that OpenMP with the static scheduling obtained the worst results with 8 processors and almost the best results with 32 processors. Given that the static scheduling distributes linearly the chunks of iterations, this version should be able to exploit the data locality. Also, one would expect a uniform access time for all processors. Looking at the tracefiles, no important imbalance was find is the static version, although some processors were executing one chunk of iterations more. Therefore, it was very surprising that although the L3 and TLB miss ratio indicators are perfectly balanced for all processors and were less than the $50 \%$ in the static version than in the dynamic version, the dynamic version was beating the static one with 8 processors.

We analysed the memory bandwidth obtained by the static and dynamic scheduling versions, which is shown in figures 18 and 19. While the static scheduling is able to achieve more or less the same bandwidth in the three cases (with 8, 16 and 32 processors), with the dynamic scheduling the bandwidth obtained by the example is each time smaller. This explains the improvement of the static version with regard the dynamic version when higher number of processors

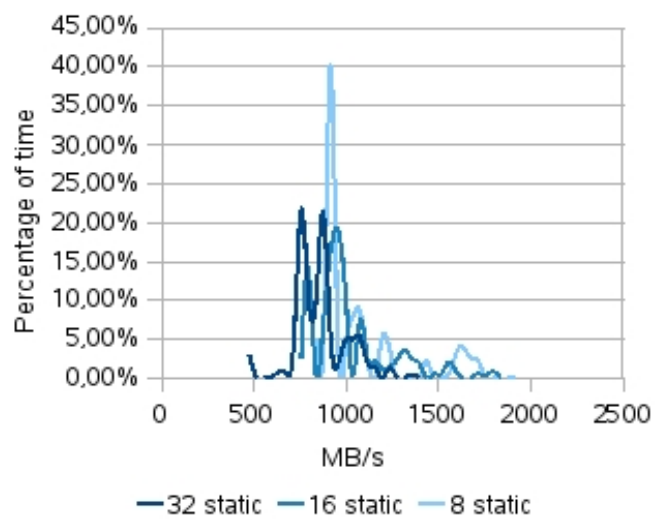

Figure 18: Bandwidth with memory for the OpenMP version when using static scheduling

is used. However, it is still not clear why the dynamic case is better than the static with 8 processors.

Since the arrays were split in chunks of data that are not multiple of the memory page size, a possible explanation would be that the first touch instantiation of a page does not necessarily guarantees that a chunk is local in the same module where the thread is running. We repeated the example with new sizes (with chunks of size multiple of the memory page size) and also with balanced load for all the processors. In this case, the behavior of the example improves significantly, as well as the bandwidth achieved when using 8 processors as can be seen in figure 20. The peak of the curve is now centered on $1220 \mathrm{MB} / \mathrm{s}$. Since the tracefiles with only one processor per node show that the peak bandwidth achievable with this example is $5000 \mathrm{MB} / \mathrm{s}$, we can not expect much more with 4 processors per node. Additionally, if all threads in the same node access memory at the same time they create conflicts between them. 


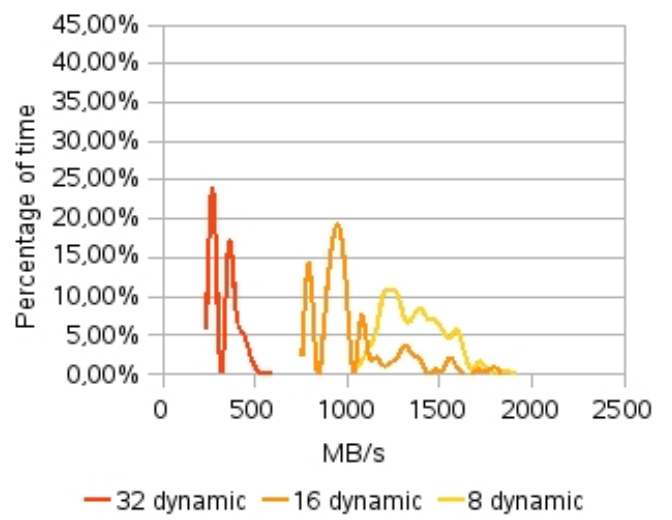

Figure 19: Bandwidth with memory for the OpenMP version when using dynamic scheduling

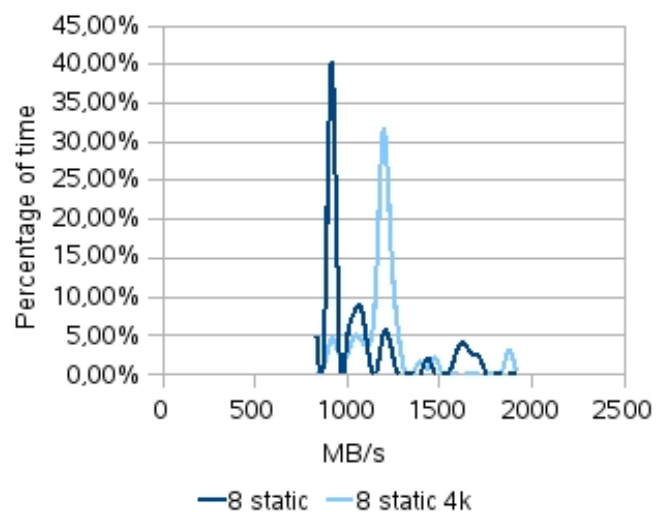

Figure 20: Bandwidth with memory for the OpenMP version when using static scheduling (impact of using data size multiple of memory page)

\section{Further exploiting the memory locality}

As seen in the previous section, exploiting the memory locality in ccNUMA based systems has a large impact in the performance results and that a dynamic scheduling conscious of the locality should be able to obtain good performance. With the objective of further improving how SMPSs exploits the data locality we have made a modification in the way way tasks are assigned to the threads.

The existing mechanism in SMPSs exploits the locality by executing sequences of data dependent tasks in the same thread. However, the creation of these sequences of tasks can be interrupted either by the SMPSs runtime graph-creation mechanism (that has a threshold on the maximum number of tasks in the graph for memory allocation reasons) or by

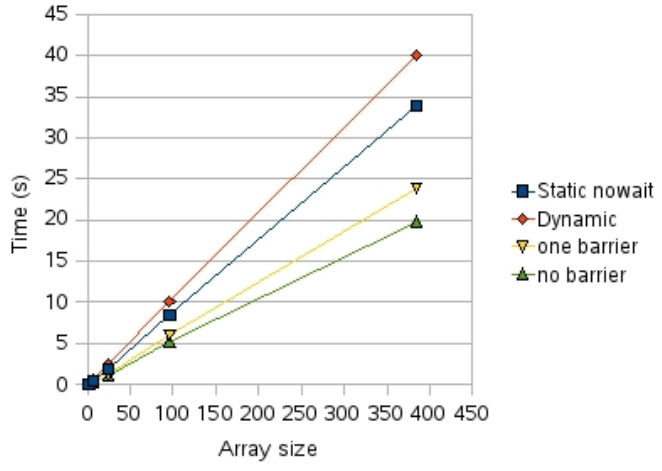

Figure 21: Execution time of the STREAM benchmark with when changing array size ( 8 processors)

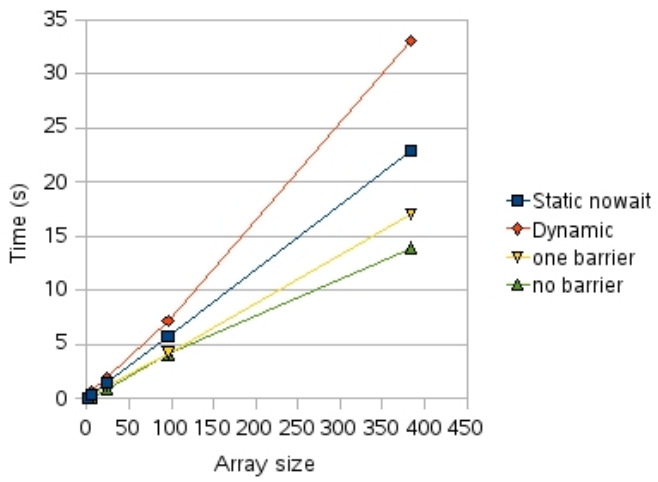

Figure 22: Execution time of the STREAM benchmark with when changing array size (16 processors)

the program synchronization points (i.e. barriers).

The new implemented mechanism is able to remember wich thread did the first touch of a given block of data. Whenever a new task is added by the SMPSs runtime, if this task is ready, instead of inserting it in the main ready list is directly inserted (in FIFO order) in the ready list of the thread that first touched the data accessed by the task.

Figures 21, 22, and 23 compare a new set of versions, both for OpenMP and for SMPSs. First, the size of the arrays' chunks is now a multiple of a memory page size in all cases. Also, the number of chunks by wich we divide the array is a multiple of the number of processors. Additionally, for the static scheduling version, the "nowait" clause is added in the pragmas. However, this clause can not be inserted when dynamic scheduling is used, since OpenMP does not preserve the data dependences and the benchmark does not validates. For the SMPSs versions, we changes as well the sizes of the arrays and chunks of data. Two versions are tested, which use the mechanism described above to map tasks to threads: one that performs the initialization of the data (in parallel) and a barrier is inserted after this 


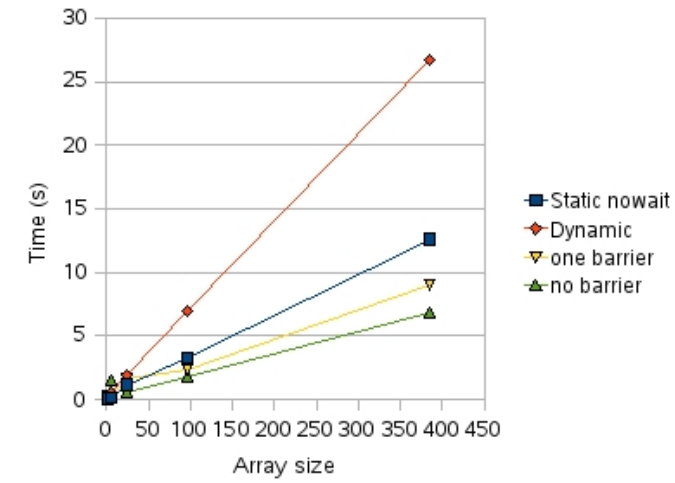

Figure 23: Execution time of the STREAM benchmark with when changing array size (32 processors)

initialization and a second one that does not insert any barrier.

A significant difference here is that the OpenMP version with static scheduling and the nowait clause outperforms the dynamic scheduling in all cases. Also, SMPSs versions with the new memory affinity mechanism, show better performance than the OpenMP cases for all processor counts.

\section{Conclusions}

The paper presents a comparison of how the benchmark STREAM can be implemented with OpenMP and with SMPSs and how the different scheduling mechanisms impact in the achievable memory bandwidth. With OpenMP, an evaluation of the impact in the performance in a memory intensive benchmark of the static and dynamic scheduling is presented. With SMPSs, results obtained with different versions inserting barriers and without inserting them is presented. For both OpenMP and SMPSs, the impact of initializing the data sequentially is analyzed and clearly for ccNUMA memory based systems this is a non-appropriate option since this will allocate all the data in the same memory module, creating a bottle-neck in the memory accesses.

Besides, both OpenMP and SMPSs require scheduling schemes that although conscious of the locality (and this is somehow a quite static feature) are dynamic enough to adapt to other sources of imbalance in the systems. More specific for SMPSs, increasing the threshold of number of tasks in the graph would enable to better exploit the temporal locality that appears in computations far away in the original source code.

\section{Acknowledgments}

The authors acknowledge the financial support of the Comision Interministerial de Ciencia y Tecnologa (CICYT,
Contract TIN2007-60625) and the BSC-IBM MareIncognito research agreement.

\section{References}

[1] cOMPunity. The community of OpenMP users, researchers, tool developers and provider website. http://www.compunity.org/, 2006.

[2] E. Ayguadé, N. Copty, A. Duran, J. Hoeflinger, Y. Lin, and G. Zhang. A proposal for task parallelism in OpenMP. In Proceedings of the 3rd International Workshop on OpenMP, June 2006.

[3] J.M. Perez, R.M. Badia, and J.Labarta. A dependencyaware task-based programming environment for multi-core architectures. In Proceedings of IEEE Cluster Computing 2008, 2008.

[4] E. Ayguadé, A. Duran, J. Hoeflinger, F. Massaioli, and $X$. Teruel. An experimental evaluation of the new openmp tasking model. In Proceedings of the 20th International Workshop on Languages and Compilers for Parallel Computing, 2007.

[5] J. M. Perez, P. Bellens, R. M. Badia, and J. Labarta. CellSs: Programming the Cell/B.E. made easier. IBM Journal of Research and Development, 51(5), Aug 2007.

[6] M. Frigo, C. E. Leiserson, and K. H. Randall. The implementation of the cilk-5 multithreaded language. SIGPLAN Notices, 33(5):212-223, 1998.

[7] M. Gonzalez, E. Ayguadé, X. Martorell, and J. Labarta. Exploiting pipelined executions in OpenMP. In Proceedings of the 32nd Annual International Conference on Parallel Processing, pages 153-160, Oct 2003.

[8] A. Duran, J.M. Perez, E. Ayguade, R.M. Badia, and J. Labarta. Extending the OpenMP tasking model to allow dependent tasks. In Proceedings of the 4th International Workshop on OpenMP, 2008.

[9] G. Juckeland, M.S. Muller, W.E. Nagel, and S Pflu. Accessing data on sgi altix: An experience with reality. In Proceedings of WMPI 2006, 2006.

[10] A. Kayi, E. Kornkven, T. El-Ghazawi, and G. Newby. Application performance tuning for clusters with ccnuma nodes. In Computational Science and Engineering, 2008. CSE '08. 11th IEEE International Conference on, pages 245-252, July 2008.

[11] R. Ferrer, M. González, F. Silla, X. Martorell, and E. Ayguadé. Evaluation of memory performance on the cell be with the sarc programming model. In Proceedings of MEDEA workshop (PACT), 2008.

[12] HPCS. The hpc challenge benchmark. http://icl.cs.utk.edu/hpcc/index.html.

[13] Jesús Labarta, Sergi Girona, Vincent Pillet, Toni Cortes, and Luis Gregoris. DiP: A parallel program development environment. In Proceedings of the 2nd International EuroPar Conference (EuroPar 96), 1996. 